\title{
Be stars in open clusters
}

\section{Balmer line spectroscopy ${ }^{\star}$}

\author{
J.M. Torrejón ${ }^{1}$, J. Fabregat ${ }^{1}$, G. Bernabeu ${ }^{2}$, and S. Alba ${ }^{1}$ \\ 1 Departamento de Astronomía y Astrofísica, Universidad de Valencia, 46100 Burjassot, Valencia, Spain \\ 2 Departamento de Ingeniería de Sistemas y Comunicaciones, Universidad de Alicante, Apdo. 99, 03080 Alicante, Spain
}

Received August 2; accepted November 26, 1996

\begin{abstract}
We present $\mathrm{H} \alpha$ line profiles $\left(R \simeq 310^{3}\right.$, $S / N \simeq 10^{2}$ at continuum level, $\simeq 50$ for some objects) for 58 stars in 8 northern open clusters and associations, complemented with $\mathrm{H} \beta$ and $\mathrm{H} \gamma$ profiles for 36 of them as well as $\mathrm{H} \delta$ and $\mathrm{H} \epsilon$ for 16 programme stars. Our observations, though lower in resolution, yield results which are in good agreement with those obtained in earlier works based mainly on field Be stars. Our data suggest a similar spectroscopic behavior for both field and cluster Be stars.

The line parameters (EW, $\left.F W H M, \Delta v_{\mathrm{p}}\right)$ have been obtained for each line. Nearly the whole set of stars have previously published quasi-simultaneous uvby $\beta$ photometry, so a study of reliable correlations between photometric and spectroscopic data can be undertaken.
\end{abstract}

Key words: lines: profiles - stars: emission line, be open clusters and associations: general

\section{Introduction}

Be objects are defined as stars of spectral type B, and luminosity classes III to V, whose spectra show, or have shown at some time, Balmer (and sometimes other) lines in emission. The origin of this emission is atomic transitions in a circumstellar envelope, relatively cool $\left(\sim 10^{4} \mathrm{~K}\right)$ and relatively dense $\left(\sim 10^{11} \mathrm{~cm}^{-3}\right)$ with a composition similar to that of the stellar photosphere. The contribution of the circumstellar envelope has dramatic effects upon the observables: the slope of the continuum is distorted,

Send offprint requests to: J.M. Torrejon

jmt@castor.matapl.uv.es

* Based on observations made at the German-Spanish Astronomical Center, Calar Alto, Spain which is operated by the Max Planck Institut für Astronomie, Heidelberg, jointly with the spanish National Commission for Astronomy. the photospheric absorption lines are filled-in with an unknown amount of circumstellar emission in such a way that it is very difficult to deduce either the spectral type of the underlying star or the actual amount of the emitting material, the line profiles themselves are distorted and the photometric indices contaminated. As a consequence we cannot apply the usual photometric and spectroscopic techniques to derive the astrophysical parameters of the underlying star.

Our goal is to elaborate a method to determine the intrinsic parameters of Be stars from uvby $\beta$ photometry and Balmer line spectroscopy. A first attempt to develop such a method was the work of Fabregat \& Reglero (1990) based on published studies of emission properties of circumstellar disks around Be stars. However the photometry and the spectroscopy were not simultaneous. Since Be stars are known to be highly variable some uncertainty is expected in the derived relationships. Thus, the latter authors consider their calibration to be preliminary.

With this in mind we planned to build a set of stars with high quality photometry and spectroscopy, as simultaneous as possible and with accurate values of reddening and distance modulus. This latter requirement was fulfilled by observing Be stars with well established membership of open clusters so that both interstellar reddening and distance modulus can be inferred from that of the cluster (Mermilliod 1982; Slettebak 1985). In Fabregat et al. (1996), hereafter called Paper I, we presented the photometric measurements along with a careful analysis of the data. One of the main results obtained was the observation of a clearly different photometric behavior between Be stars earlier than B5V and later ones. This fact, of course, is expected to contribute to the uncertainty of the above mentioned calibration.

In the present paper we present the spectroscopy and analyze the data as far as the available resolution makes it 
possible, mainly in order to guarantee the homogeneity of the sample. Further work will include the elaboration of a calibration valid for Be type stars, the determination of astrophysical parameters for circumstellar envelopes and their modeling.

\section{The observations and reduction procedure}

We carried out two observing runs during the months of December 1992 and August 1993, both with the Cassegrain spectrograph attached to the $2.2 \mathrm{~m}$ telescope at the Calar Alto Observatory, Almería, Spain. The detector used in both runs was the GEC\#15 CCD. The two instrumental arrangements were different, giving slightly different resolutions. For the first configuration, we used the $f / 3$ camera, at $0.66 \AA /$ pixel spectral dispersion, giving a resolution of about $1.5 \AA$ at $\lambda 6000 \AA$. For the latter arrangement we used the $f / 1.5$ camera which produces a spectral dispersion $1.36 \AA$ pixel $^{-1}$ giving a resolution of about $2.7 \AA$, at $\lambda 6000 \AA$. The red spectra were centered on the $\mathrm{H} \alpha$ line at $6563 \AA$. The blue spectra included only $\mathrm{H} \beta(4861 \AA)$ and $\mathrm{H} \gamma(4340 \AA)$ in the first run, while in the second one we extended the range from $\mathrm{H} \beta$ to the Balmer discontinuity ( $3647 \AA$ ).

The spectra were reduced by means of the Starlink FIGARO software package (Shortridge 1991). Several bias frames were taken during the night, their stability was checked and then they were averaged, before subtraction from the program frames. No drift in dark current was found after several hours of operation, and at different time exposures. The flat fields were recorded at the end of the night. A smooth function was fitted to the flat field spectrum with which each program frame was normalized. Finally, wavelength calibration was done by comparison with available He-Ar calibration lamps at the telescope using the corresponding subroutine provided by FIGARO. As much as twenty different lines were identified and used for calibration purposes.

The measurement of equivalent widths, full widths at half maximum, and velocity peak separation for the Balmer lines was done with the Starlink DIPSO package (Howarth \& Murray 1991). Sufficient continuum was taken into account to allow the wings to effectively reach the continuum level. For stars where the Balmer jump was recorded, we measured the EW only up to $\mathrm{H} \epsilon$. Beyond this point the continuum was poorly defined by line blocking and the merging of the wings, although it can be seen up to H15 or more. Full widths at half maximum were measured only for emission lines.

An accurate treatment of the errors in the estimation of equivalent widths is very difficult. The main source of uncertainty is the exact location of the continuum, which for a poor signal-to-noise ratio can reach an uncertainty of up to $10 \%$ (Gray 1992). In order to estimate a mean error for our measurements, we decided to accurately study some selected spectra following the method outlined in Chalabaev \& Maillard (1983). The result was that the estimation by this method is completely consistent with the statistical dispersion when several measurements, assuming several continuum levels, are made with DIPSO. As a general rule we can assign an absolute error of about $1 \AA$. On the other hand, large emission often implies very extended wings which can cause an overestimation of the continuum level of up to $10 \%$ and hence underestimation of the EW. These contributions to measurement errors have to be added quadratically so that, roughly speaking, a maximum error of about $10 \%$ can be assigned to all the measurements.

No correction has been applied to equivalent widths to account for photospheric absorption since our goal in the present paper is just to present the spectroscopic observations and extract some conclusions without the assumption of any model. In fact, the correction will be done in the future using the photometric data of Paper I. However, corrections for instrumental broadening have been applied to full widths by means of the usual relation

$F W H M=\left[(F W H M)_{\mathrm{obs}}^{2}-(F W H M)_{\text {inst }}^{2}\right]^{\frac{1}{2}}$

where $(F W H M)_{\text {inst }} \sim 2 \AA$, for the first run and around $2.7 \AA$, for the second. Mean estimated errors are probably around $\pm 50 \mathrm{~km} / \mathrm{s}$.

Rotational velocities have been taken from Slettebak $(1982,1985)$. All these results are shown in Tables 1 to 3, while in Table 4 we present the observed equivalent widths for regular B type stars also included in our survey.

\section{The data}

\subsection{An atlas of hydrogen lines}

Measured line profiles are plotted in Figs. 1 through 14 with continua normalized to unity for 58 programme stars in 8 open clusters and associations. $\mathrm{H} \alpha, \mathrm{H} \beta$ and $\mathrm{H} \gamma$ are shown for each star and, in separate figures, $\mathrm{H} \delta$ and $\mathrm{H} \epsilon$. Stars where only $\mathrm{H} \alpha$ was measured are shown following the first ones for each cluster. The horizontal axes (wavelength) have been drawn at the same scale for all the lines spanning $80 \AA$, so that relative changes in the line widths can easily be observed. The number in the upper right corner is the star identification number and is the same as in Paper I except for NGC 457 which are from Boden (1946). For the four stars of the Pleiades cluster for which spectra were recorded twice, the date of observations are included in the plot following the star number.

$50 \%$ of our programme stars (29 of 58) show single peak emission at $\mathrm{H} \alpha$ while $26 \%$ (15 of 58) show double 
Table 1. Equivalent widths $(\AA)$ and full widths at half maximun $(\mathrm{km} / \mathrm{s})$ for the three first Balmer lines. Full widths at half maximum have been measured only for emission lines. Rotational velocities have been taken from Slettebak (1982, 1985). Identification numbers for NGC 457 are from Boden (1946) while for the rest of stars are the same as in Paper I

\begin{tabular}{|c|c|c|c|c|c|c|c|c|c|}
\hline \multirow[t]{2}{*}{$\overline{\text { Cluster }}$} & \multirow[t]{2}{*}{ Star } & \multirow[t]{2}{*}{$W_{\alpha}$} & \multirow[t]{2}{*}{$W_{\beta}$} & \multirow[t]{2}{*}{$\overline{W_{\gamma}}$} & \multirow[t]{2}{*}{$v \sin i$} & \multicolumn{3}{|c|}{$F W H M$} & \multirow[t]{2}{*}{ rem. } \\
\hline & & & & & & $\mathrm{H} \alpha$ & $\mathrm{H} \beta$ & $\mathrm{H} \gamma$ & \\
\hline \multirow[t]{4}{*}{ NGC 457} & 14 & -5.9 & 0.6 & 2.1 & & 148 & 175 & & \\
\hline & 91 & -9.5 & 1.6 & 2.7 & & 571 & & & \\
\hline & 153 & -44.1 & -4.7 & -1.1 & & 286 & 288 & 213 & \\
\hline & 198 & -2.0 & 2.0 & 3.1 & & 455 & & & \\
\hline \multirow[t]{20}{*}{ NGC 663} & 2 & -43.0 & & & & 392 & & & \\
\hline & 6 & -33.5 & & & & 157 & & & \\
\hline & 8 & -11.0 & & & & 401 & & & \\
\hline & 10 & -10.7 & & & 250 & 350 & & & \\
\hline & 21 & -4.0 & 2.4 & 2.8 & 100 & 314 & & & \\
\hline & 51 & -8.7 & & & & 572 & & & \\
\hline & 84 & -42.7 & & & & 325 & & & \\
\hline & 92 & -1.9 & 2.7 & 3.0 & 200 & 450 & & & \\
\hline & 93 & -48.0 & & & 150 & 246 & & & \\
\hline & 107 & 2.4 & & & 100 & & & & \\
\hline & 110 & 2.5 & & & 80 & & & & \\
\hline & 121 & -21.0 & & & & 400 & & & \\
\hline & 124 & -14.6 & & & 200 & 380 & & & \\
\hline & 130 & -32.4 & & & & 399 & & & \\
\hline & 141 & -44.0 & -3.3 & 1.5 & 250 & 305 & 274 & 173 & \\
\hline & 194 & -16.5 & -1.1 & 1.5 & 250 & 400 & 334 & 112 & 1 \\
\hline & 222 & -16.3 & & & 80 & 181 & & & \\
\hline & 224 & -37.0 & & & & 380 & & & \\
\hline & 243 & -8.6 & 1.6 & 3.0 & 100 & 324 & 261 & & \\
\hline & 297 & -37.0 & & & 250 & 349 & & & \\
\hline \multirow[t]{13}{*}{$\mathrm{h} \& \chi$ Per } & 146 & 3.1 & 2.8 & 3.3 & 200 & & & & 2 \\
\hline & 309 & -34.3 & -3.5 & 0.4 & 270 & 268 & 219 & 227 & \\
\hline & 566 & 1.8 & & & 250 & & & & 2 \\
\hline & 717 & -1.4 & 1.8 & 3.1 & & 166 & 112 & & \\
\hline & 1261 & -58.4 & -4.7 & 0.4 & 300 & 375 & 272 & 301 & \\
\hline & 1268 & 2.3 & 2.5 & 2.8 & & & & & \\
\hline & 1702 & -20.9 & -1.4 & 2.3 & 150 & 194 & 168 & 141 & \\
\hline & 2088 & -10.6 & 0.3 & 2.6 & 180 & 441 & 438 & & \\
\hline & 2138 & -2.1 & 1.4 & 2.6 & 100 & 268 & & & \\
\hline & 2165 & -27.2 & -2.6 & 1.7 & 100 & 147 & 92 & 90 & \\
\hline & 2284 & -66.4 & -5.3 & -0.1 & 300 & 263 & 218 & 235 & \\
\hline & 2371 & 1.1 & 2.2 & 2.8 & 100 & & & & \\
\hline & 2402 & -8.1 & -0.1 & 1.9 & 150 & 404 & 530 & & \\
\hline \multirow{4}{*}{$\begin{array}{l}\text { Pleiades } \\
\text { (Dec. 92) }\end{array}$} & 486 & 4.2 & 6.3 & 6.0 & 180 & & & & \\
\hline & 980 & -11.7 & 3.7 & 5.0 & 280 & 340 & 424 & & \\
\hline & 1432 & -4.0 & 4.7 & 4.9 & 140 & 211 & & & 3 \\
\hline & 2181 & -29.8 & 2.8 & 5.8 & 320 & 327 & 269 & & 4 \\
\hline \multirow{4}{*}{$\begin{array}{l}\text { Pleiades } \\
\text { (Aug. 93) }\end{array}$} & 486 & 4.0 & 6.0 & 6.7 & 180 & & & & \\
\hline & 980 & -11.2 & 3.9 & 5.9 & 280 & 342 & 357 & & \\
\hline & 1432 & & 4.6 & 5.8 & 140 & & & & 3 \\
\hline & 2181 & -36.5 & 2.1 & 6.4 & 320 & 314 & 306 & & 4 \\
\hline NGC 2323 & 157 & -3.7 & 6.1 & 5.8 & & 175 & 134 & & 5 \\
\hline \multirow[t]{3}{*}{ NGC 2422} & 42 & -5.8 & 6.4 & 7.5 & & 98 & 118 & & \\
\hline & 45 & -26.2 & -1.2 & 1.9 & 230 & 452 & 337 & & 5 \\
\hline & 125 & -6.4 & 5.0 & 5.6 & 275 & 269 & & & \\
\hline
\end{tabular}


Table 1. continued

\begin{tabular}{|c|c|c|c|c|c|c|c|c|c|}
\hline \multirow[t]{2}{*}{ Cluster } & \multirow[t]{2}{*}{ Star } & \multirow[t]{2}{*}{$W_{\alpha}$} & \multirow[t]{2}{*}{$W_{\beta}$} & \multirow[t]{2}{*}{$\overline{W_{\gamma}}$} & \multirow[t]{2}{*}{$v \sin i$} & \multicolumn{3}{|c|}{ FWHM } & \multirow[t]{2}{*}{ rem. } \\
\hline & & & & & & $\mathrm{H} \alpha$ & $\mathrm{H} \beta$ & $\mathrm{H} \gamma$ & \\
\hline \multirow[t]{4}{*}{ NGC 7654} & 778 & & 3.9 & 4.7 & & & 417 & & 5 \\
\hline & 782 & -2.2 & 4.9 & 4.4 & & 213 & & & 5 \\
\hline & 928 & -8.3 & 5.4 & 6.7 & & 434 & & & 5 \\
\hline & 989 & -27.5 & & & & 343 & & & 5 \\
\hline \multirow[t]{9}{*}{ Cep OB3 } & 6 & 10.3 & & & & & & & 2 \\
\hline & 9 & 4.6 & & & & & & & 2 \\
\hline & 15 & -17.6 & 1.7 & 3.4 & & 384 & 324 & & 6 \\
\hline & 40 & 2.8 & 3.4 & 4.0 & & & & & \\
\hline & 51 & 9.6 & 11.4 & 10.0 & & & & & 2 \\
\hline & 64 & 9.0 & & & & & & & 2 \\
\hline & 65 & 8.9 & & & & & & & 2 \\
\hline & 69 & -1.8 & 2.6 & 3.4 & & 279 & & & 7 \\
\hline & 80 & 10.5 & & & & & & & 2 \\
\hline
\end{tabular}

1.- Be/X ray binary RX J0146.9+6121 (LSI +61 235).

2.- Non member (Paper I).

3.- Blue straggler. Mermilliod (1982a).

4.- Be-Shell star.

5.- Variable reddening across cluster face.

6.- Herbig Ae/Be type object.

7.- Eclipsing binary CW Cep.

peaks. The remaining 14 stars show absorption lines. No shell type profiles have been detected in our sample.

We have decided to include lines in absorption as well because: 1) they all belong to stars previously classified as Be and it might be useful to compare them with previous ones in an emission phase, 2) the absorption lines seen in some stars, for example 1268 and 2371 in $h \& \chi$ Per, have equivalent widths smaller than those for a normal $\mathrm{B}$ star $(3.5-10 \AA)$, denoting the presence of some emission filling-in, although no emission can be seen from the inspection of the $\mathrm{H} \alpha$ line profile.

As stated before, and following the line of Paper I, our main purpose here is to present the spectroscopic data along with a first analysis in order to guarantee their homogeneity. Thus we are going to test the quality of the data by applying some previously found relationships based on large samples of Be stars and higher resolution spectroscopy.

\subsection{Relationship between equivalent widths and full widths at half maximum}

It was found by Dachs et al. (1986) that the full width at half maximum of an emission line and its equivalent width are inversely correlated by a law of the form

$F W H M(\mathrm{H} \alpha) / 2=\left(W_{\alpha} /-3 \AA\right)^{-\frac{1}{4}}(v \sin i+$ const. $)$.

The anticorrelation between $W_{\alpha}$ and $F W H M(\mathrm{H} \alpha)$ is in the sense that the higher the emission, the narrower the line. This is consistent with the picture in which large emission corresponds to large disk radius, thus slow velocity and small rotational broadening.

In order to check whether this relation holds for our data we have followed the method outlined by Hanuschik et al. (1988) and have written the equation in the form

$\log (F W H M(\mathrm{H} \alpha) / 2 v \sin i)=a \log \left(W_{\alpha} / \AA\right)+b$.

These values are plotted in Fig. 15 and the best least squares fit yields:
$a=-0.235 \pm 0.065$
$b=0.176 \pm 0.138$
$r=0.60$ 
where $r$ is the correlation coefficient as usual. These values are consistent with those obtained by Dachs et al. (1986) and Hanuschik et al. (1988). Considering only early type stars, in the sense established in Paper I, the parameters are:

$a=-0.284 \pm 0.057$

$b=0.274 \pm 0.109$

$r=0.79$

where both $\sigma$ and $r$ are distinctly better, while for late Be type stars there seems to be no correlation at all.

We also checked this method with $\mathrm{H} \beta$ and obtained the following results:

$a=-0.301 \pm 0.083$

$b=-0.182 \pm 0.053$

$r=0.76$

For $\mathrm{H} \gamma$ the few points available do not allow us to reach any conclusion.

\subsection{Relationship between full widths at half maximum and $v \sin i$}

It is known that a trend exists in the full widths at half maximum of the hydrogen series lines in the sense that $F W H M(H n)$ is equal or narrower than $F W H M(H n+1)$. This agrees with the model whereby higher members of the series are produced in more rapidly rotating regions of the circumstellar disk closer to the star, and are thus more affected by rotational broadening.

In Fig. 16 we have plotted the measured full width at half maximum for emission lines against published values of projected rotational velocity $v \sin i$ (Slettebak 1982, 1985).

We have computed the relation:

$<F W H M\left(H_{n}\right) / v \sin i>$

for $n=\alpha, \beta, \gamma$. It should be noted that the $F W H M$ of those lines showing double peaks is roughly twice that presented by single lines. The former, therefore show high values of $\left(F W H M\left(H_{n}\right) / v \sin i\right)$. Taking this into account, the parameters obtained are the following:

\begin{tabular}{rrrr}
\hline lines & $\alpha$ & $\beta$ & $\gamma$ \\
\hline all & $1.76 \pm 0.69$ & $1.38 \pm 0.82$ & $0.79 \pm 0.18$ \\
single & $1.41 \pm 0.37$ & $0.99 \pm 0.19$ & $0.79 \pm 0.17$ \\
\hline
\end{tabular}

These values are somewhat higher than those obtained by Slettebak et al. (1992). Those authors, apart from a larger sample of stars which improves the statistics, used corrected values of $F W H M$ for emission profiles by subtracting computed photospheric lines taking into account rotational broadening, shape distortion, gravity darkening and change of spectral type due to rotation and then extrapolating mathematically the remaining emission profile. Our purpose in this paper, however, is to deliberately avoid imposing any kind of model.

Table 2. Equivalent widths $(\AA)$ for $\mathrm{H} \delta$ and $\mathrm{H} \epsilon$. Remarks are the same as in Table 1

\begin{tabular}{lrrrr}
\hline Cluster & Star & $W_{\delta}$ & $W_{\epsilon}$ & rem. \\
\hline NGC 457 & 14 & 2.1 & 2.9 & \\
& 91 & 3.1 & 4.2 & \\
& 153 & 0.9 & 1.9 & \\
& 198 & 2.9 & 3.8 & \\
NGC 663 & 21 & 2.8 & 3.8 & \\
& 92 & 2.9 & 3.8 & \\
& 141 & 2.2 & 3.2 & \\
& 194 & 2.2 & 3.0 & 1 \\
& 243 & 3.0 & 3.6 & \\
Pleiades & 486 & 6.5 & 6.8 & \\
(Aug. 93) & 980 & 5.5 & 5.8 & \\
& 1432 & 5.4 & 5.7 & 3 \\
& 2181 & 6.7 & 6.6 & 4 \\
NGC 7654 & 778 & 5.6 & 6.2 & 5 \\
& 782 & 5.4 & 6.0 & 5 \\
& 928 & 2.6 & 7.0 & 5 \\
\hline
\end{tabular}

These values, as well as those given in the preceding Section, are all consistent with those presented in similar discussions of earlier works based on higher resolution spectroscopy of large samples of Be field stars. We can interpret this fact in the sense that the spectroscopic behavior of Be stars in clusters is, within the limits of our resolution, similar to that presented by field Be stars.

\subsection{Relationship between $\Delta v_{\text {peak }}$ and $v \sin i$}

According to Huang (1972), a rotating envelope with velocity $v \sin i$ and outer radius $r_{\mathrm{d}}$, surrounding a star of radius $R_{\star}$, is expected to produce double peaked lines whose separation $\Delta v_{\mathrm{p}}$, is given by

$\Delta v_{\mathrm{p}} / 2 v \sin i=\left(r_{\mathrm{d}} / R_{\star}\right)^{-j}$

with $j=0.5$ for Keplerian rotation and $j=1$ for angular momentum conservation.

In Table 3 we present measured values of the velocity peak separations for those stars showing double peaks. 
The mean velocity peak separation ratio for the first two Balmer lines is:

$\Delta v_{\mathrm{p}}^{\beta} / \Delta v_{\mathrm{p}}^{\alpha}=1.78 \pm 0.38$

fully compatible with the result obtained by Hanuschik et al. (1988), and slightly above that presented by Dachs et al. (1992). The relation between velocity peak separations and corresponding values of $v \sin i$ yields $\left\langle\Delta v_{\mathrm{p}}^{\alpha}\right\rangle=$ $(1.2 \pm 0.4) v \sin i$ for $\mathrm{H} \alpha$ and $\left\langle\Delta v_{\mathrm{p}}^{\beta}\right\rangle=(1.3 \pm 0.4) v \sin i$ for $\mathrm{H} \beta$. These values differ somewhat from those given by Hanuschik et al. (1988) and the correlation here is in the sense that larger peak separations correspond to larger values of $v \sin i$. This supports the axial symmetry of the emitting envelope being $\Delta v_{\mathrm{p}}^{\beta}$ systematically larger than $\Delta v_{\mathrm{p}}^{\alpha}$ which, according to Eq. (5), means that $\mathrm{H} \beta$ emission originates closer to the star.

Table 3. Velocity peak separation $(\mathrm{km} / \mathrm{s})$

\begin{tabular}{lrrr}
\hline Cluster & Star & $\Delta v_{\mathrm{p}}^{\alpha}$ & $\Delta v_{\mathrm{p}}^{\beta}$ \\
\hline NGC 457 & 91 & 260 & 670 \\
& 198 & 260 & \\
NGC 663 & 8 & 250 & \\
& 21 & 180 & 260 \\
& 51 & 290 & \\
& 92 & 320 & \\
& 121 & 130 & \\
& 124 & 190 & \\
& 130 & 190 & \\
& 243 & & 250 \\
& & & \\
h \& $\chi$ Per & 2088 & 150 & 260 \\
& 2138 & 130 & 210 \\
& 2402 & 160 & 250 \\
Pleiades & 486 & 280 & \\
(Dec. 92) & 980 & 130 & 220 \\
& & & \\
Pleiades & 486 & 250 & \\
(Aug. 93) & 980 & 120 & 190 \\
& & & \\
NGC 2422 & 45 & & 170 \\
& 125 & & 220 \\
NGC 7654 & 778 & & 170 \\
& 928 & 250 & \\
\hline
\end{tabular}

We also checked the relationship between peak separation in velocity units and equivalent widths using the equation

$\log \left(\Delta v_{\mathrm{p}}^{\alpha} / 2 v \sin i\right)=a \log \left(W_{\alpha} / \AA\right)+b$
$a=-0.40 \pm 0.16$
$b=0.03 \pm 0.15$
$r=0.74$

These values agree with those given by Hanuschik et al. (1988) and are plotted in Fig. 17 . As stated in their work, this inverse correlation can be explained in terms of a velocity field decreasing with increasing radius of the emitting envelope. Our data would indicate some preference for values of $j \approx 0.8$ (Eq. (8b) of that reference). We did not try to check this correlation for $\mathrm{H} \beta$ since, as stated before, the equivalent widths have been measured from the continuum and the emission is almost always embedded in the broad wings of the underlying photospheric absorption. This prevent us to consider the $W_{\beta}$ as an effective measure of the amount of circumstellar emission.

\subsection{Comments on some individual stars}

Some stars from the Pleiades cluster were observed twice, thereby allowing some discussion of their variability.

The star $\mathrm{Hz} 486$ showed a significant augmentation of the emission level which is reflected in slightly shallower lines. On the other hand some enhancement appears for the $\mathrm{H} \beta$ wings. See Fig. 7 .

The star $\mathrm{Hz} 980$ maintains its emission level but clearly shows a $V / R$ inversion. The star changes from $V / R>1$ to $V / R<1$ visible both in $\mathrm{H} \alpha$ and $\mathrm{H} \beta$ (Fig. 7).

A change in the core of $\mathrm{H} \beta$ line of the star $\mathrm{Hz} 1432$ can clearly be seen. Unfortunately we could not take the corresponding $\mathrm{H} \alpha$ spectrum. See Fig. 7.

The well known Be-Shell star Hz 2181 (Pleione, B78IV-Ve) shows here an augmentation of the emission level. As was shown in Paper I, despite the high emission level observed in its spectrum, the position in the photometric diagrams is not abnormal. This effect can be observed in Slettebak (1985) for the $U B V$ colors too. Therefore, some mechanism working in this star, makes their photometric colors to mimic those for a regular B type star. There may however be another explanation: early Be stars move towards redder values in the $M_{V}-(b-y)_{0}$ plane and to bluer colors in $M_{V}-c_{0}$ plane while late type Be stars, beyond $\mathrm{B} 5 \mathrm{~V}$, remain in their usual positions despite the emission strength, as was shown in Paper I. Recently, Cramer et al. (1995) have pointed out the difficulty of distinguish this star from other normal stars by means of photometric diagrams. 
The Be nature of two stars in the cluster NGC 663 is still controversial. W 107 was observed by Merrill \& Burwell (1949) with mild emission in the interval 1945 - 1948. However, no emission was observed by Schild \& Romanishin (1976) nor by Sanduleak $(1979,1991)$ during his survey in the interval 1946 - 1990. Sanduleak (1979) suggest a possible identification error in the Merrill \& Burwell (1949) catalogue. W 110 was reported as showing weak emission $\left(W_{\alpha}=-2.4 \AA\right)$ by Schild \& Romanishin (1976), but this observation was not confirmed by Schild (1978). Sanduleak $(1979,1991)$ never observed this star in emission during his survey spanning between 1946 and 1990. In our spectra both stars present their Balmer lines in absorption, though its equivalent width is well outside the limits for normal B type stars which would imply some amount of undetectable emission filling in the photospheric absorption line. Thus we can conclude that both stars are indeed Be stars, usually showing low levels of activity, which would explain the lack of detection in prism objective surveys like that conducted by Sanduleak.

In addition to the stars previously classified as Be, we have included in our spectroscopic survey some stars in which line emission has not previously been detected, but which several authors marked as possible Be stars. Among them we have found a new Be star, Oo 717 (BD +56 502) in the $h$ Persei cluster (Fabregat et al. 1994b). This star was signaled as a possible Be by Waelkens et al. (1990) on the basis of its photometric variability. In Fig. 6 we can see its $\mathrm{H} \alpha$ line clearly in emission, with an equivalent width of $-1.4 \AA$. It should be noted that this star was observed in September 1990 by Kaufer et al. (1994). In the Kaufer et al. spectrum the $\mathrm{H} \alpha$ line was observed in almost pure absorption (only a slight filling-in is discernible). This means the strong emission line in Fig. 6 has developed in less than two years, indicating that the spectral characteristics of Oo 717 are highly variable. We have also observed Oo 245 and Oo 864, also marked by Waelkens et al. (1990) as suspected Be stars. Both stars present the Balmer lines in absorption.

Stars 65 and 73 in NGC 2422 are also marked as suspected Be stars by Shobbrook (1984), because in their photometric diagrams they occupy positions similar to the cluster Be stars. Our spectra, however, show their Balmer lines in absorption and in our photometric diagrams (Paper I), both stars are placed among the normal absorption line stars, and do not show the anomalous position claimed by Shobbrook.

\section{Summary and conclusions}

Spectroscopy for 58 Be stars in eight open clusters is presented with measurements of $F W H M, \Delta v_{\mathrm{p}}$ and values of $\mathrm{EW}$ from $\mathrm{H} \alpha$ to $\mathrm{H} \epsilon$. This is complemented by the contem-
Table 4. EW for regular B type stars

\begin{tabular}{lrrrr}
\hline Cluster & Star & $W_{\alpha}$ & $W_{\beta}$ & $W_{\gamma}$ \\
\hline NGC 663 & 11 & 10.3 & & \\
& 15 & 6.4 & & \\
& 25 & 4.4 & & \\
& 27 & 4.8 & & \\
& 35 & 6.8 & & \\
& 85 & 7.0 & & \\
& 91 & 2.7 & & \\
& 106 & 4.2 & & \\
& 147 & 3.0 & & \\
h \& $\chi$ Per & 245 & 2.7 & 3.1 & 3.6 \\
& 864 & 3.1 & & \\
NGC 2422 & 65 & 8.5 & 10.5 & 8.7 \\
& 68 & 8.7 & & \\
& 70 & 10.0 & 12.6 & 10.6 \\
& 71 & 4.7 & 6.2 & 5.7 \\
& 73 & 7.0 & & \\
& 83 & 5.0 & 6.4 & 5.9 \\
\hline
\end{tabular}

porary photometric data of Paper I. In order to preserve the homogeneity of the sample, a first examination of the results has been made showing a good agreement with earlier results of other authors based on higher resolution data. The spectroscopic behavior of Be stars in clusters is, within our limited resolution, similar to that of field $\mathrm{Be}$ stars. We also present, the measured EW for the regular B stars also included in our sample.

Our data are in agreement with the relation given by Dachs et al. (1986), and slightly modified by Hanuschik et al. (1988), between full widths at half maximum and equivalent widths (Eq. 2). The data are consistent as well with the previous finding of some preference for $j \approx 0.8$ (Eq. 5) therefore placing the rotation field somewhere between the cases of angular momentum conservation and keplerian, as well as with the already known fact that the higher the member of the series, the closer it arises to the central star.

Some stars will be rejected in future work: non members of clusters, members with uncertain values of reddening, Herbig Ae/Be type objects, Blue Stragglers, etc. Furthermore, we will divide the sample into early Be, late Be and Shell type objects since some differences seem to exist between their physical characteristics (Paper I, Slettebak et al. 1992).

Acknowledgements. We would like to acknowledge V. Reglero and J. Busquets for their help with the observations, and B. Payne for his careful reading of the manuscript. The useful suggestions made by the referee Dr. R. Hanuschik helped 
us to improve the presentation of the paper. We also thank the staff of the Calar Alto Observatory for the kind assistance during the observations. Software and data analysis facilities were provided by Starlink, which is founded by the UK PPARC. This research has made use of the Simbad database, operated at CDS, Strasbourg, France. S.A. acknowledges a research grant from the Conselleria d'Educació i Ciència, Generalitat Valenciana. J.M.T. acknowledges a research grant from the Instituto de Estudios Turolenses (CSIC).

\section{References}

Boden E., 1946, Uppsala Astr. Obs. Ann. 2, 1

Chalabaev A., Maillard J.P., 1983, A\&A 138, 140

Cramer N., Doazan V., Nicolet B., de la Fuente A., Barylak M., 1995, A\&A 301, 811

Dachs J., Hanuschik R., Kaiser D., Rohe D., 1986, A\&A 159, 276

Dachs J., Hummel W., Hanuschik R.W., 1992, A\&AS 95, 437

Fabregat J., Reglero V., 1990, MNRAS 247, 407

Fabregat J, Torrejón J.M., Reig P., Bernabeu G., 1994a, in: Pulsation, Rotation and Mass loss in Early-Type Stars, Proc IAU Symp 162, Balona L., Heinrich H., Lecontel J.M. (eds.). Kluwer Academic Press, Holland, p. 309

Fabregat J., Torrejón J.M., Bernabeu G., 1994b, Be Star News 29,8

Fabregat J,Torrejón J.M., Reig P., et al., 1996, A\&AS 119, 271

Gray, D.F., 1992, The observation and analysis of stellar photospheres. Cambridge University Press

Hanuschik R.W., Kozok J.R., Kaiser D., 1988, A\&A 189, 147

Howarth I.D., Murray J., 1991, SERC-RAL Starlink User Note 50.13

Kaufer A., Szeifert T., Krenzin R., Baschek B., Wolf B., 1994, A\&A 289, 740

Mermmilliod J.C., 1982, A\&A 109, 48

Merrill P.W., Burwell C.G., 1949, ApJ 110, 387

Sanduleak N., 1979, AJ 84, 1319

Sanduleak N., 1990, AJ 100, 1239

Schild R.E., 1978, ApJS 37, 77

Schild R.E., Romanishin W., 1976, ApJ 204, 493

Shobbrook R.R., 1984, MNRAS 211, 659

Slettebak A., 1982, ApJ 50, 55

Slettebak A., 1985, ApJ 59, 769

Slettebak A., Collins G.W., Truax R.J., 1992, ApJS 81, 335

Waelkens C., Lampens P., Heynderickx D., et al., 1990, A\&AS 83,11 

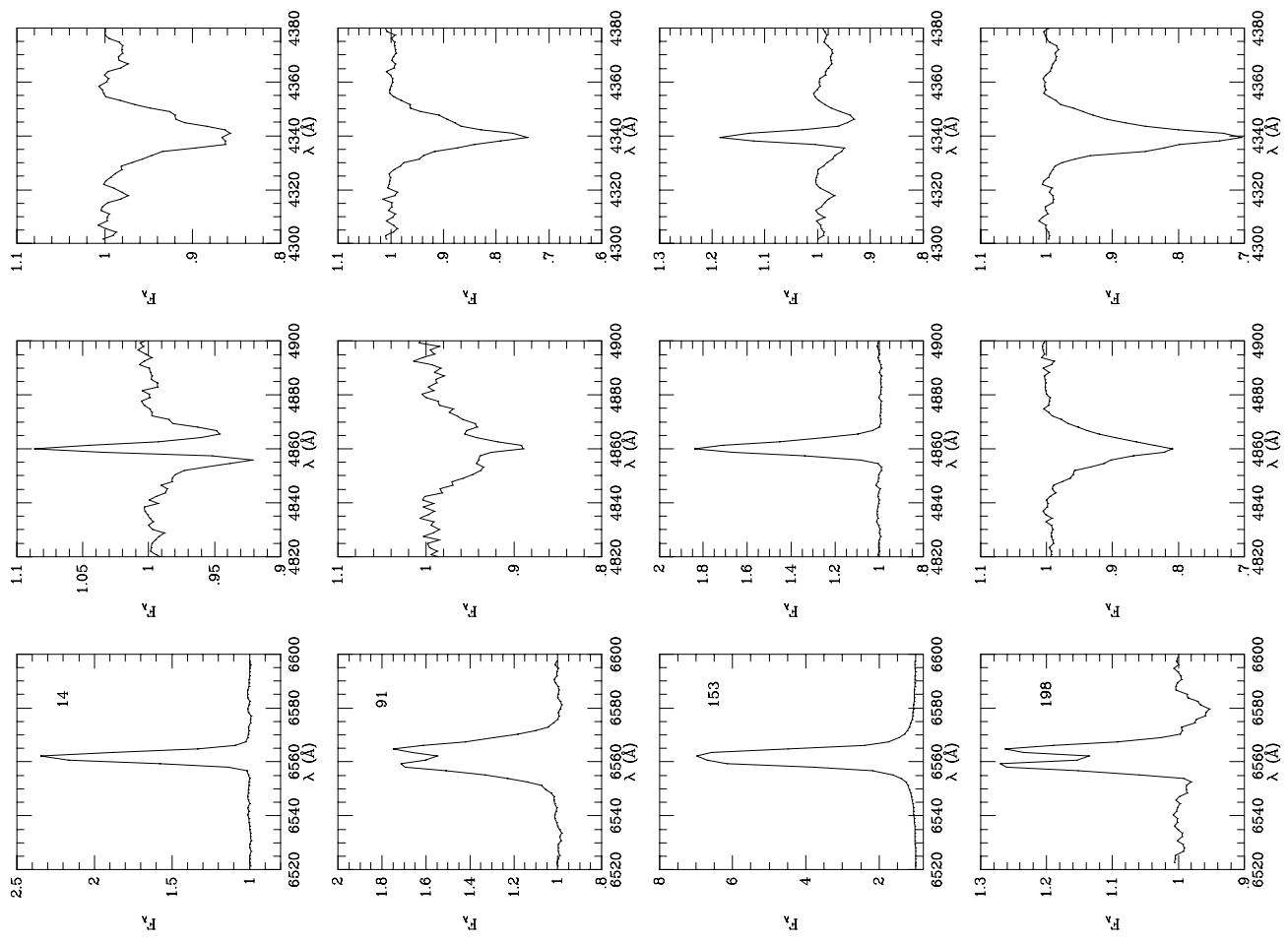

Fig. 1. $\mathrm{H} \alpha, \mathrm{H} \beta$ and $\mathrm{H} \gamma$ for stars in NGC 457. Flux has been normalized to continuum level. (To be seen in landscape)
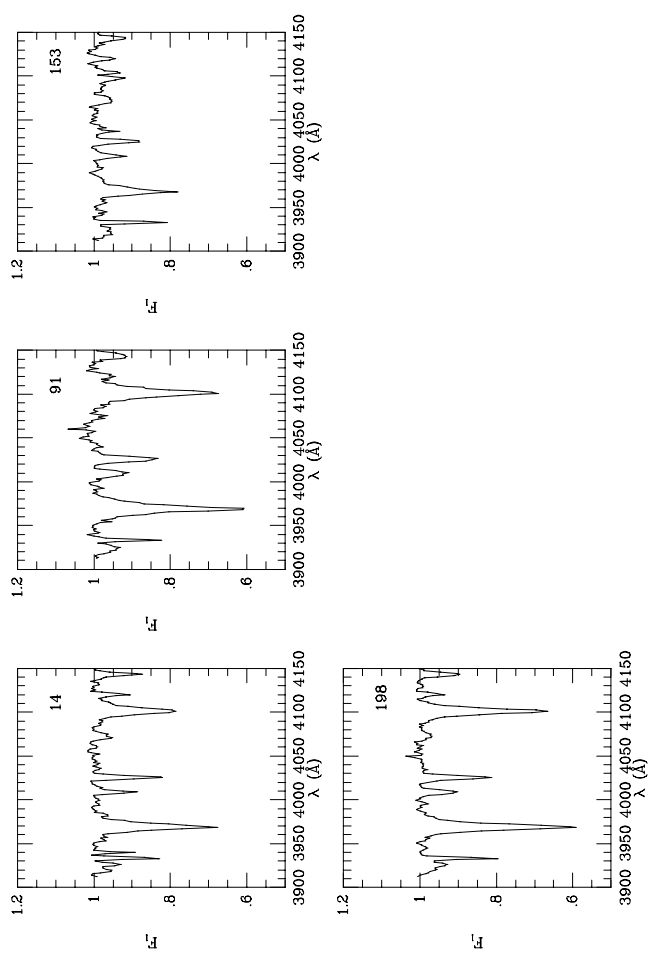

Fig. 2. $\mathrm{H} \delta$ and $\mathrm{H} \epsilon$ for stars in NGC 457. HeI is also visible. (To be seen in landscape) 

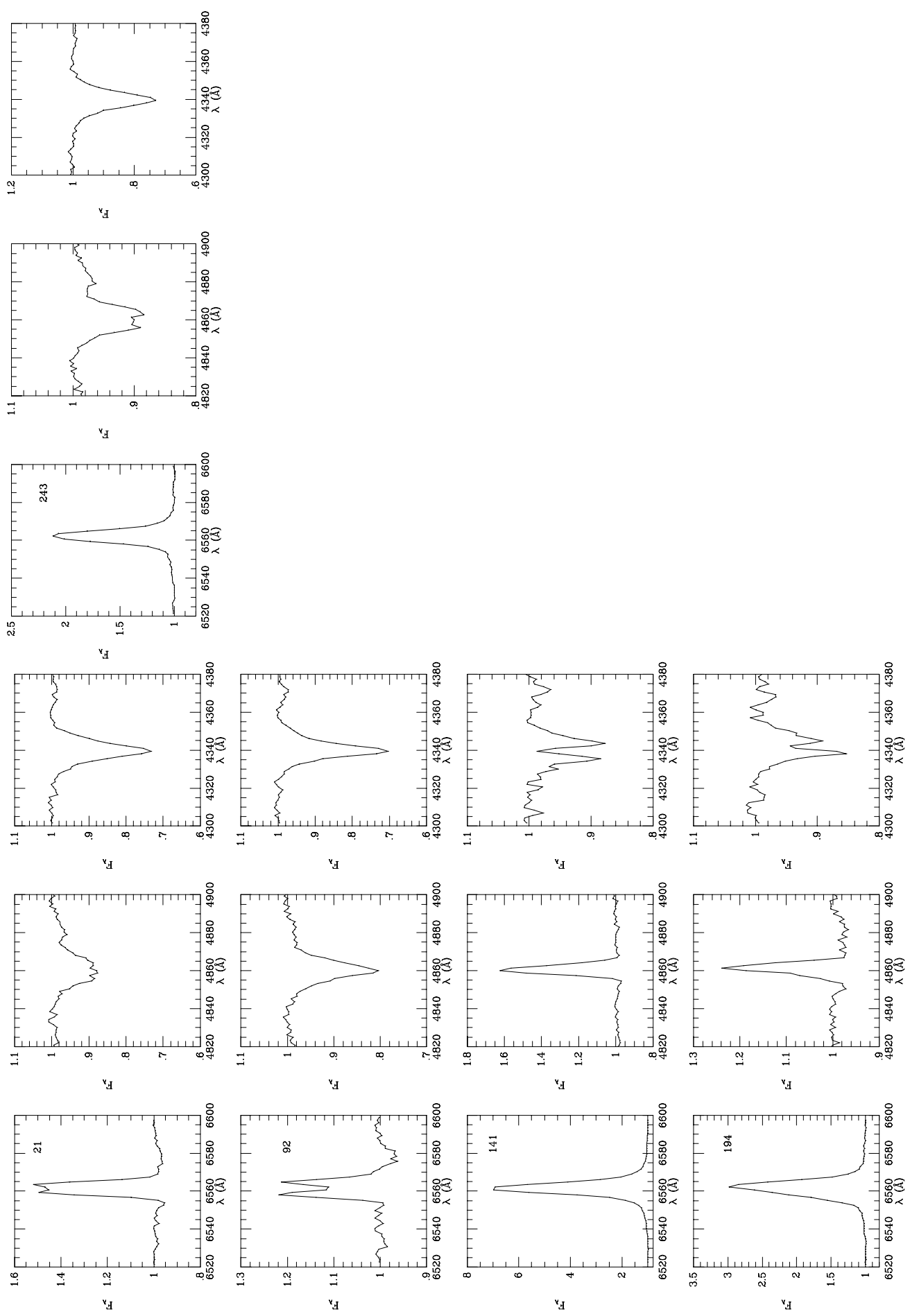

Fig. 3. a,b) $\mathrm{H} \alpha, \mathrm{H} \beta$ and $\mathrm{H} \gamma$ for stars in NGC 663. (To be seen in landscape) 

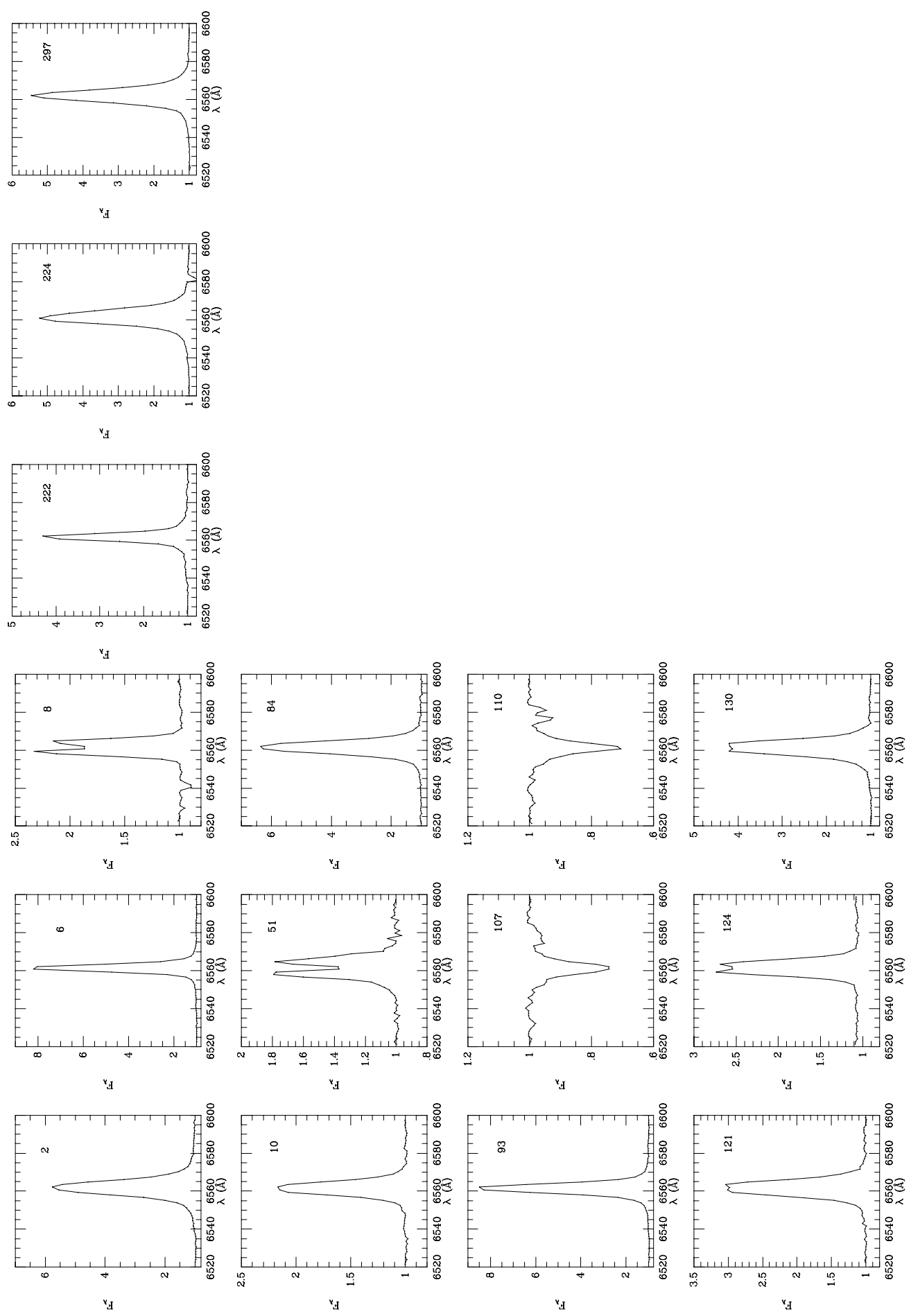

Fig. 4. a,b) $\mathrm{H} \alpha$ for stars in NGC 663. (To be seen in landscape) 

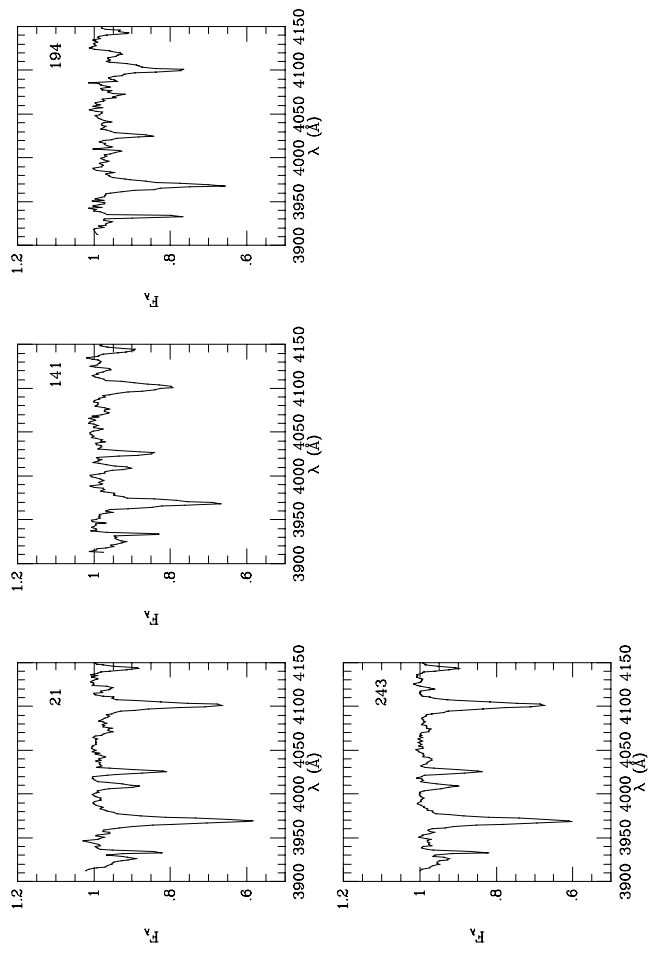

Fig. 5. $\mathrm{H} \delta$ and $\mathrm{H} \epsilon$ for stars in NGC 663. HeI is also visible. (To be seen in landscape)
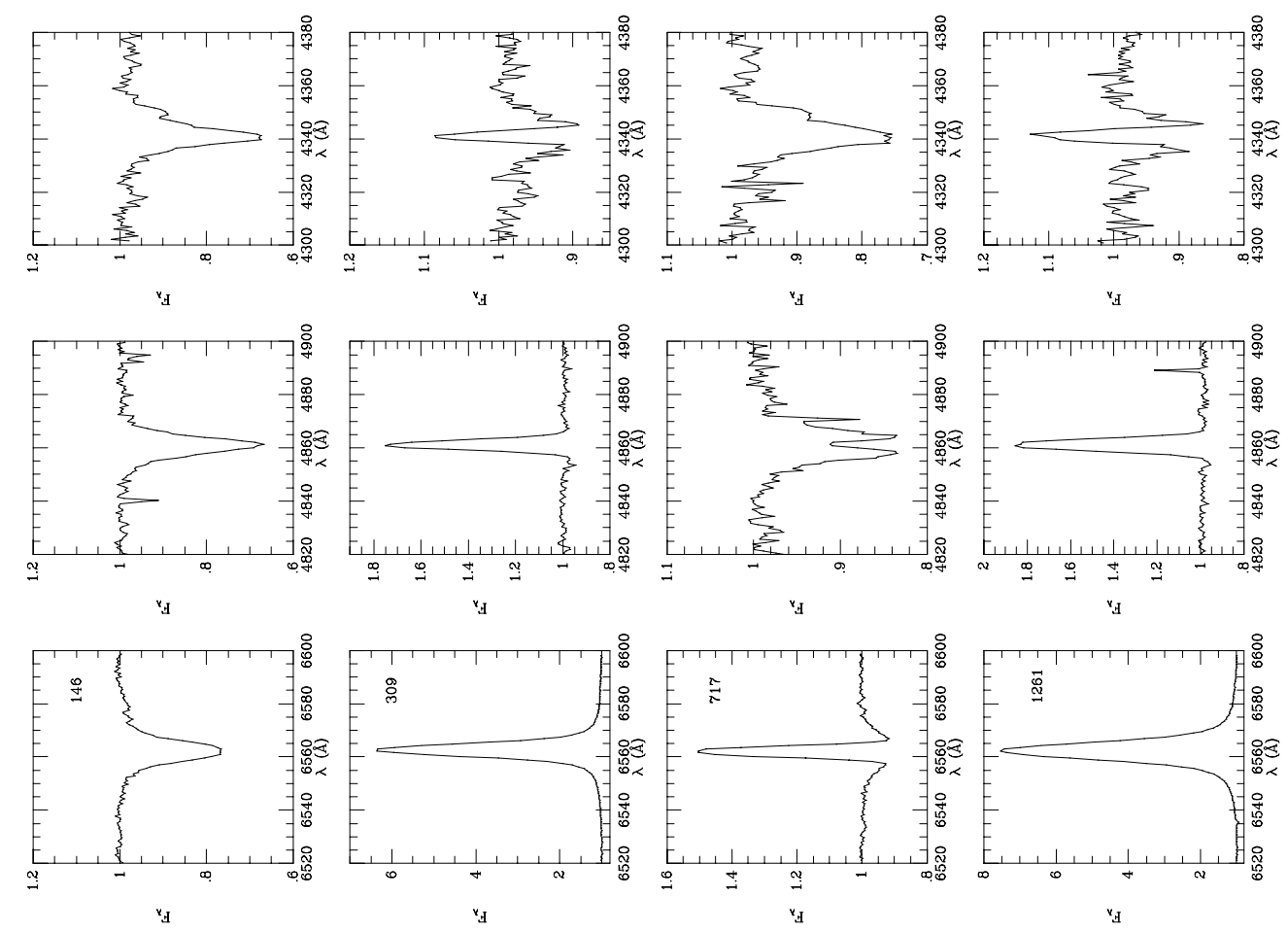

Fig. 6. $\mathrm{H} \alpha, \mathrm{H} \beta$ and $\mathrm{H} \gamma$ for $h \& \chi$ Per. (To be seen in landscape) 

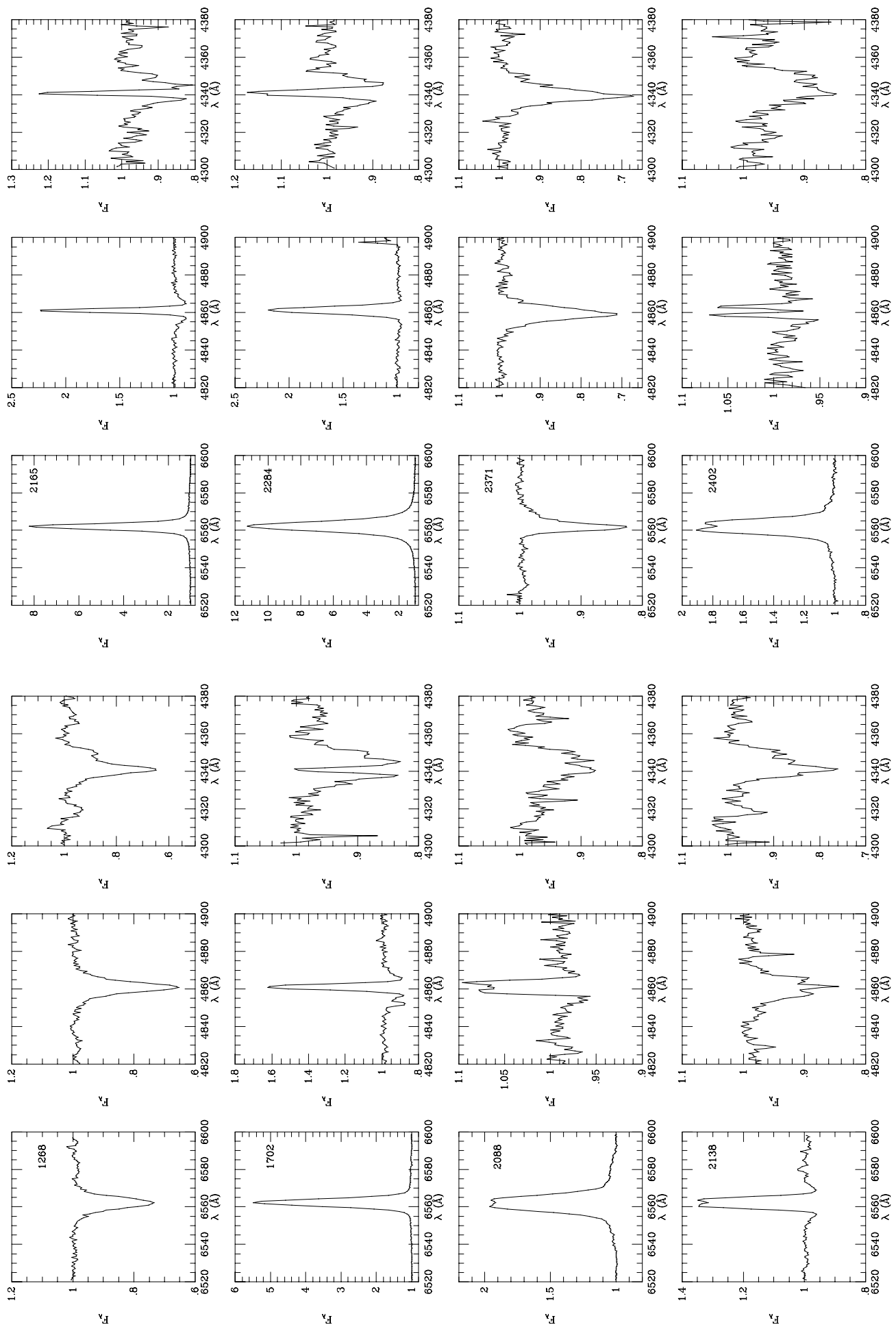

Fig. 6. continued. (To be seen in landscape) 

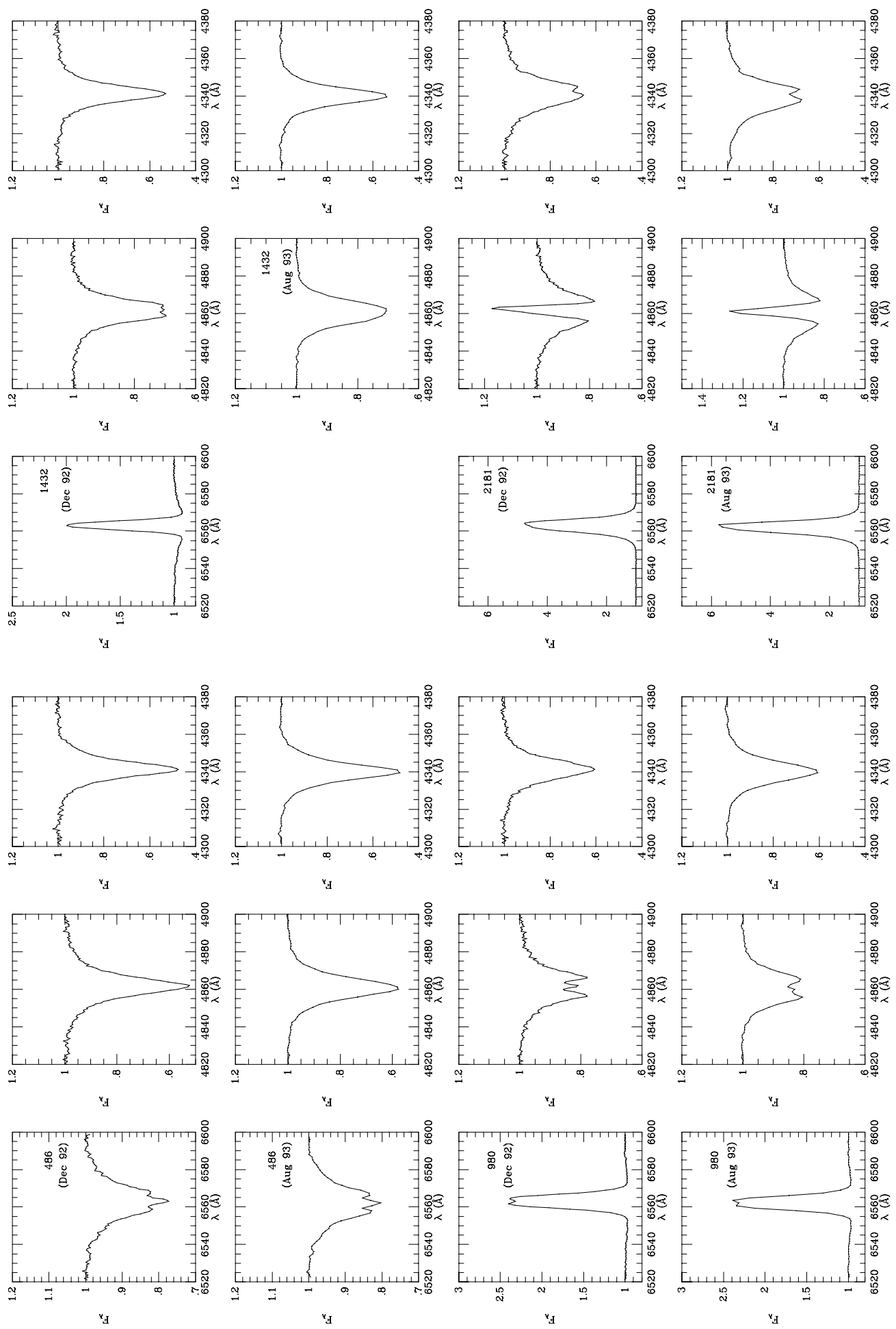

Fig. 7. $\mathrm{H} \alpha, \mathrm{H} \beta$ and $\mathrm{H} \gamma$ for Pleiades. Besides the star number, the date of observation is shown. (To be seen in landscape) 

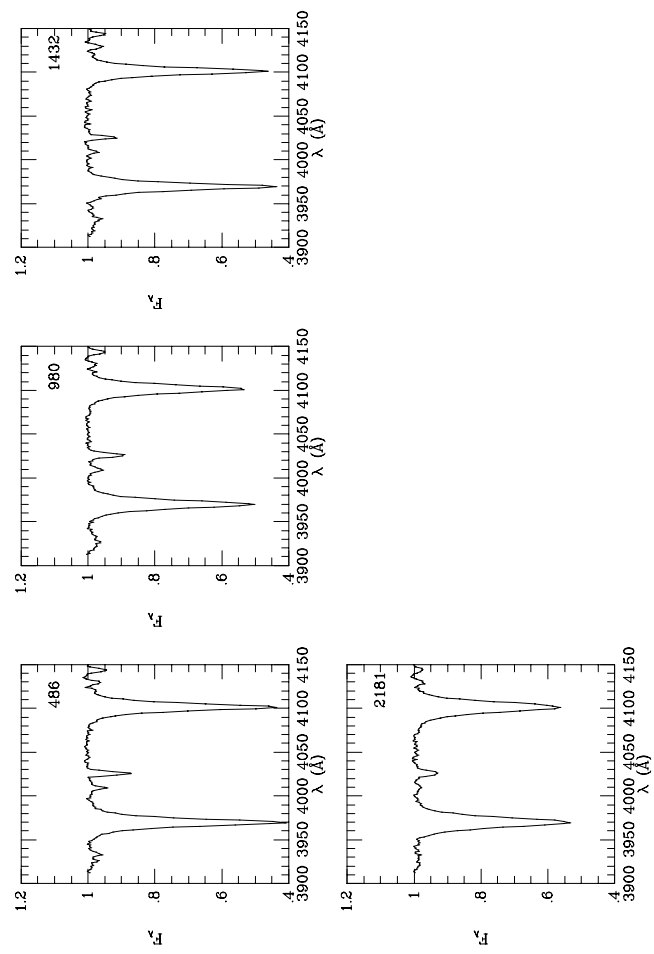

Fig. 8. $\mathrm{H} \delta$ and $\mathrm{H} \epsilon$ for Pleiades. $\mathrm{HeI}$ is also visible. (To be seen in landscape)
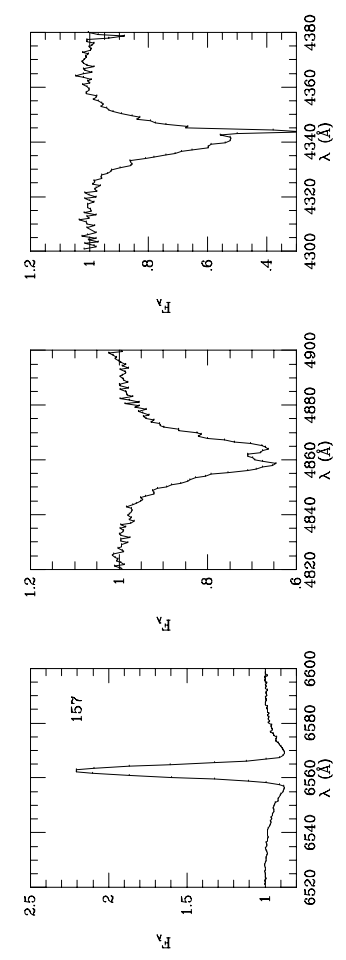

Fig. 9. $\mathrm{H} \alpha, \mathrm{H} \beta$ and $\mathrm{H} \gamma$ for star 157 in NGC 2323. (To be seen in landscape) 

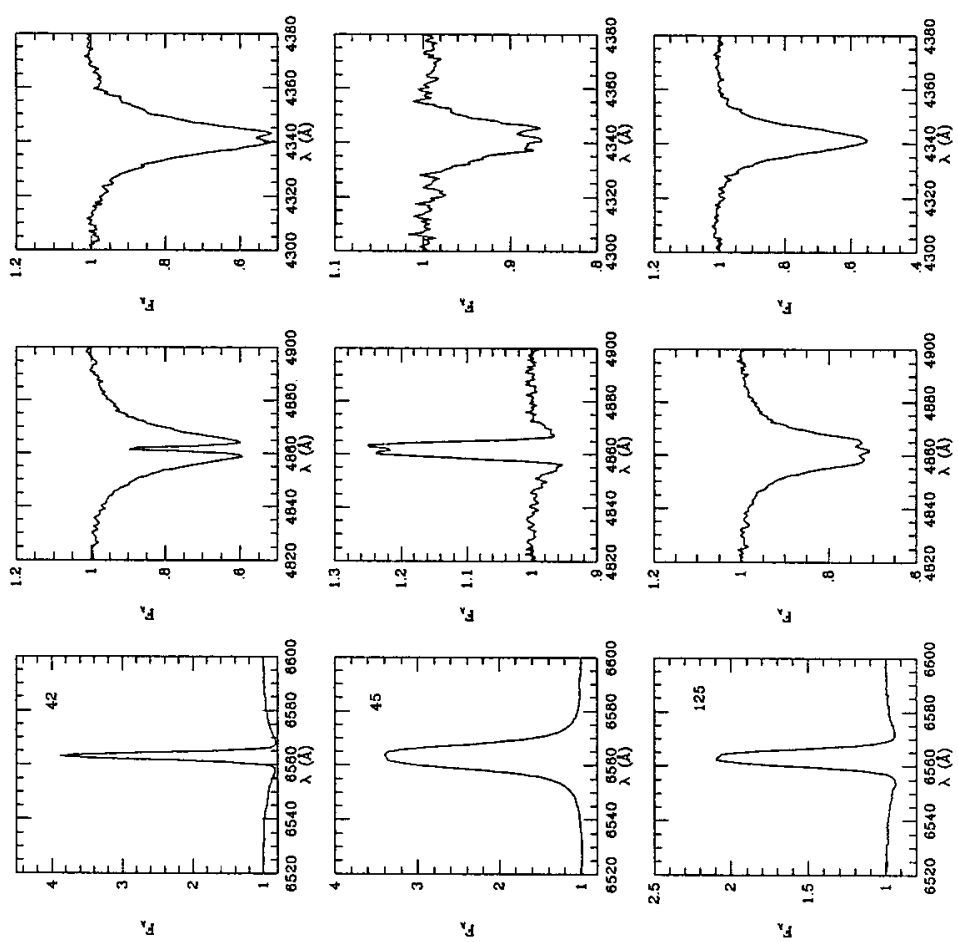

Fig. 10. $\mathrm{H} \alpha, \mathrm{H} \beta$ and $\mathrm{H} \gamma$ for stars in NGC 2422. (To be seen in landscape)
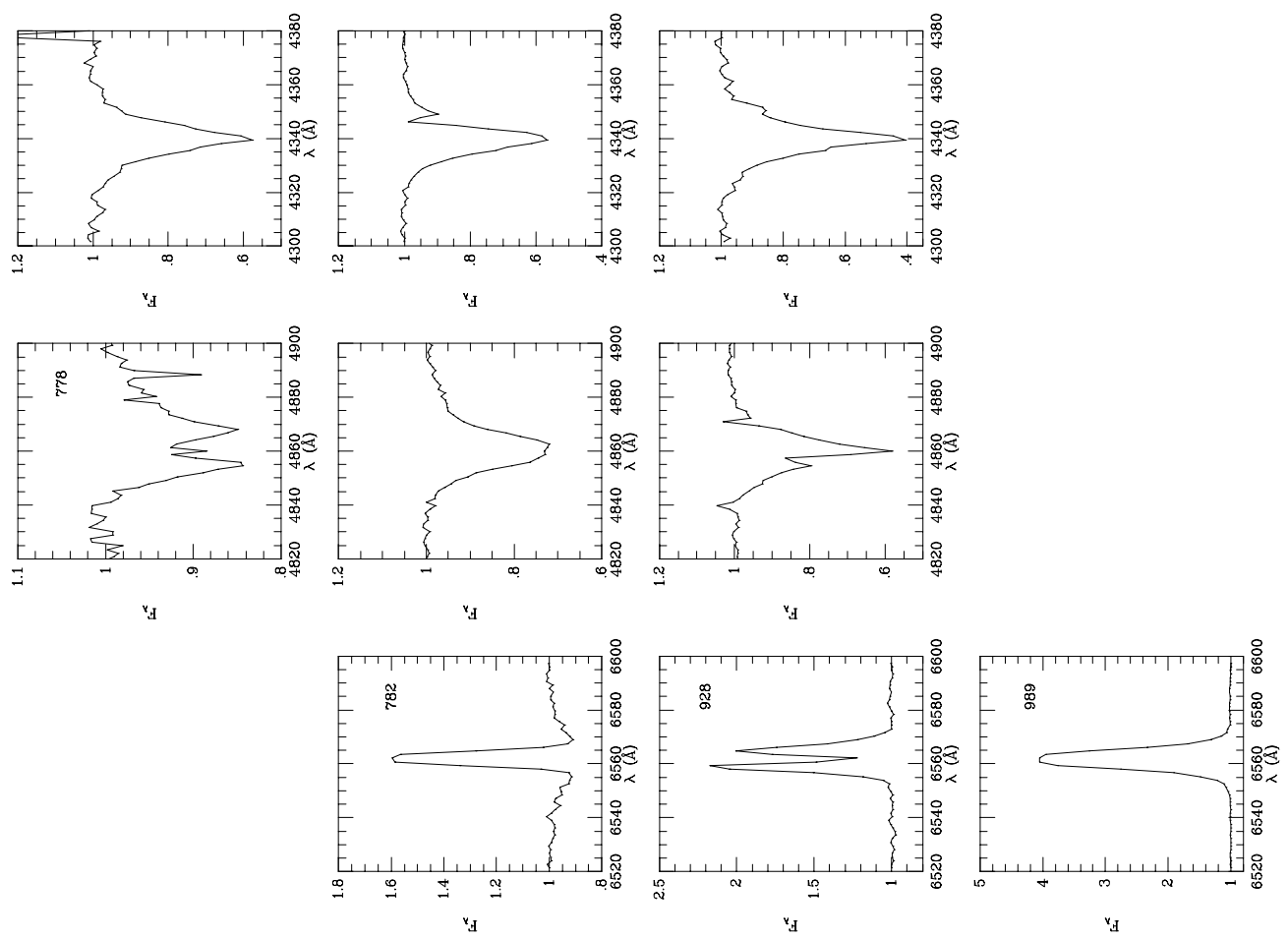

Fig. 11. $\mathrm{H} \alpha, \mathrm{H} \beta$ and $\mathrm{H} \gamma$ for stars in NGC 7654. (To be seen in landscape) 

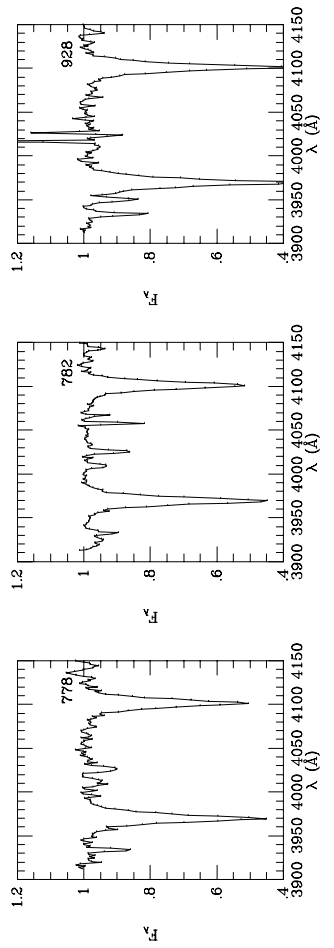

Fig. 12. $\mathrm{H} \delta$ and $\mathrm{H} \epsilon$ for stars in NGC 7654. HeI is also visible. (To be seen in landscape)
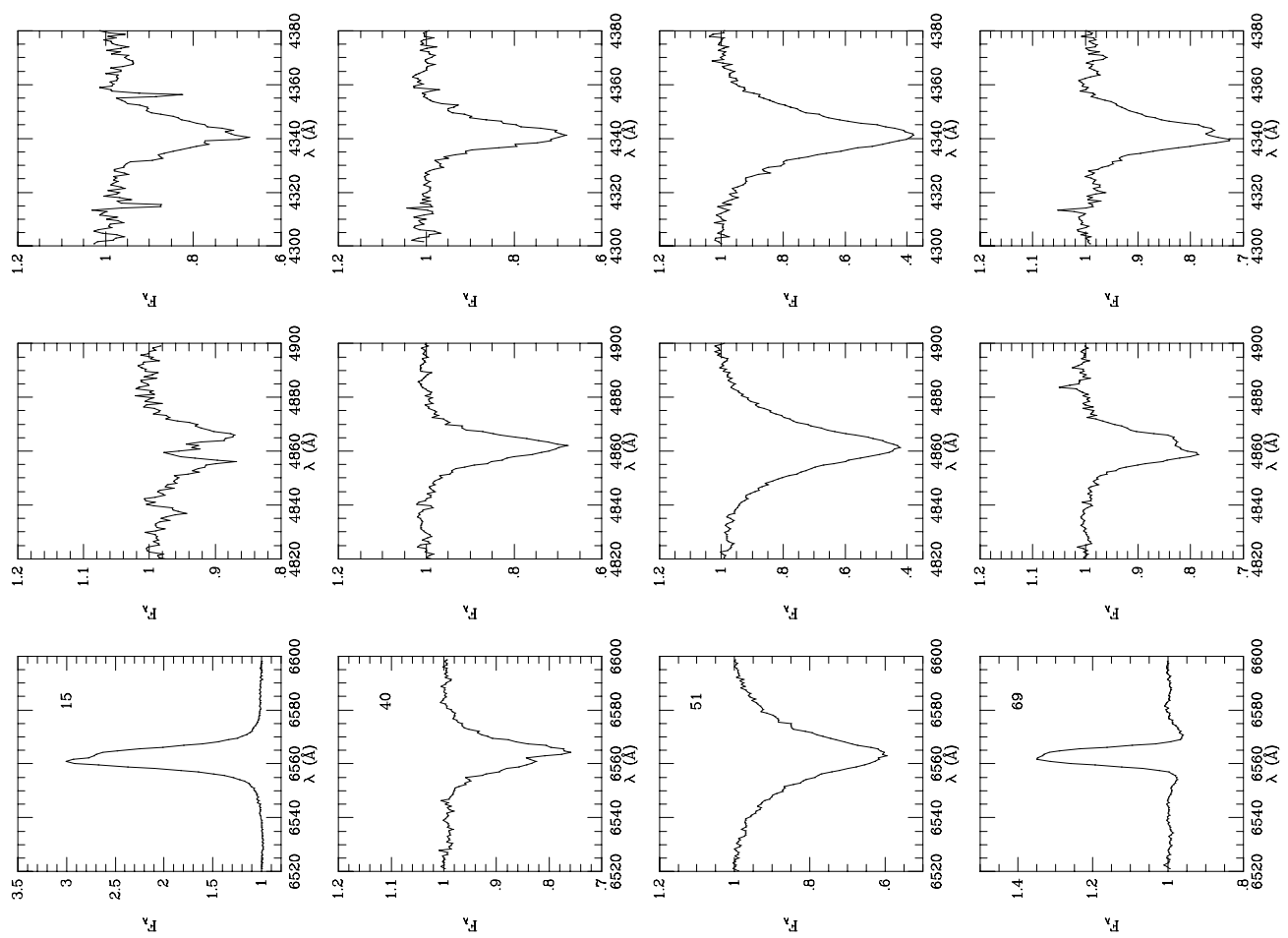

Fig. 13. $\mathrm{H} \alpha, \mathrm{H} \beta$ and $\mathrm{H} \gamma$ for stars in Cep OB3. (To be seen in landscape) 

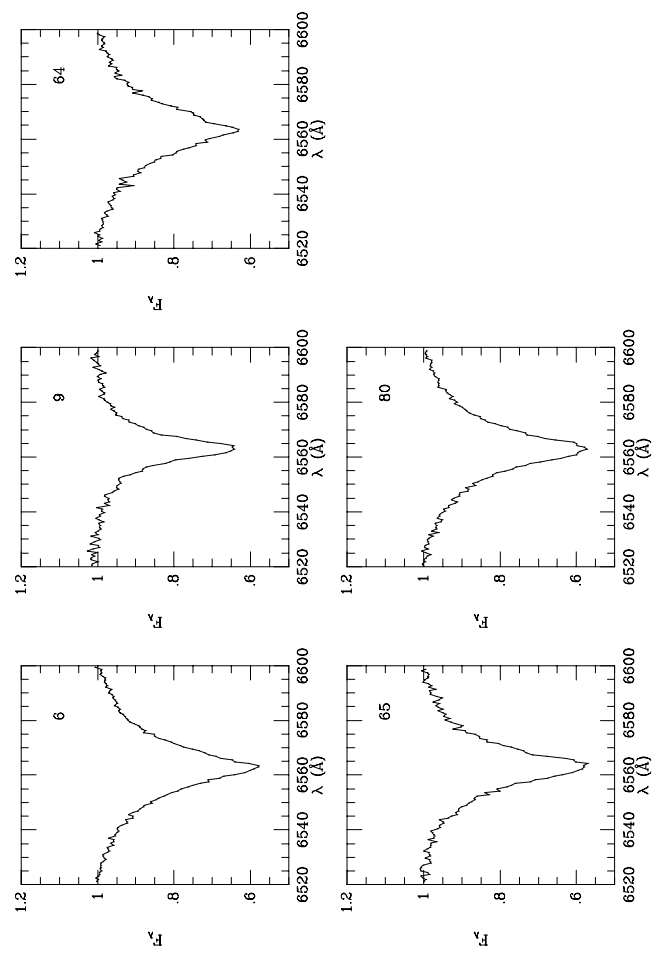

Fig. 14. $\mathrm{H} \alpha$ for stars in Cep OB3. (To be seen in landscape)
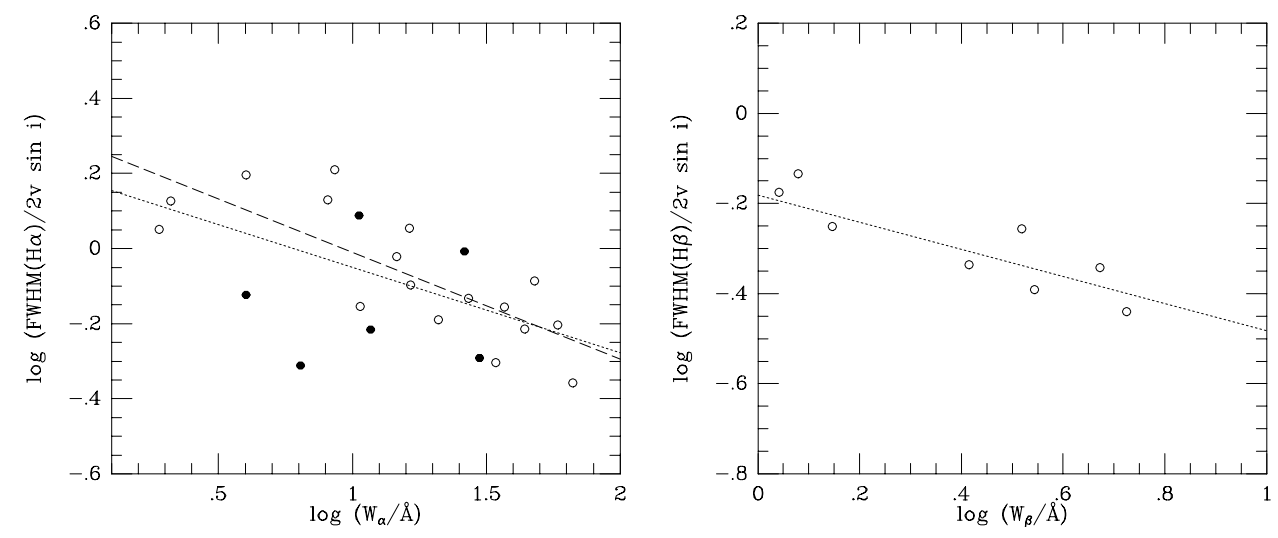

Fig. 15. Full width at half maximun for emission lines vs. equivalent width. Dotted line and dashed line represent the relationships for the whole sample and the early type Be stars respectively. Open circles are early type Be stars and solid circles are late type Be stars 

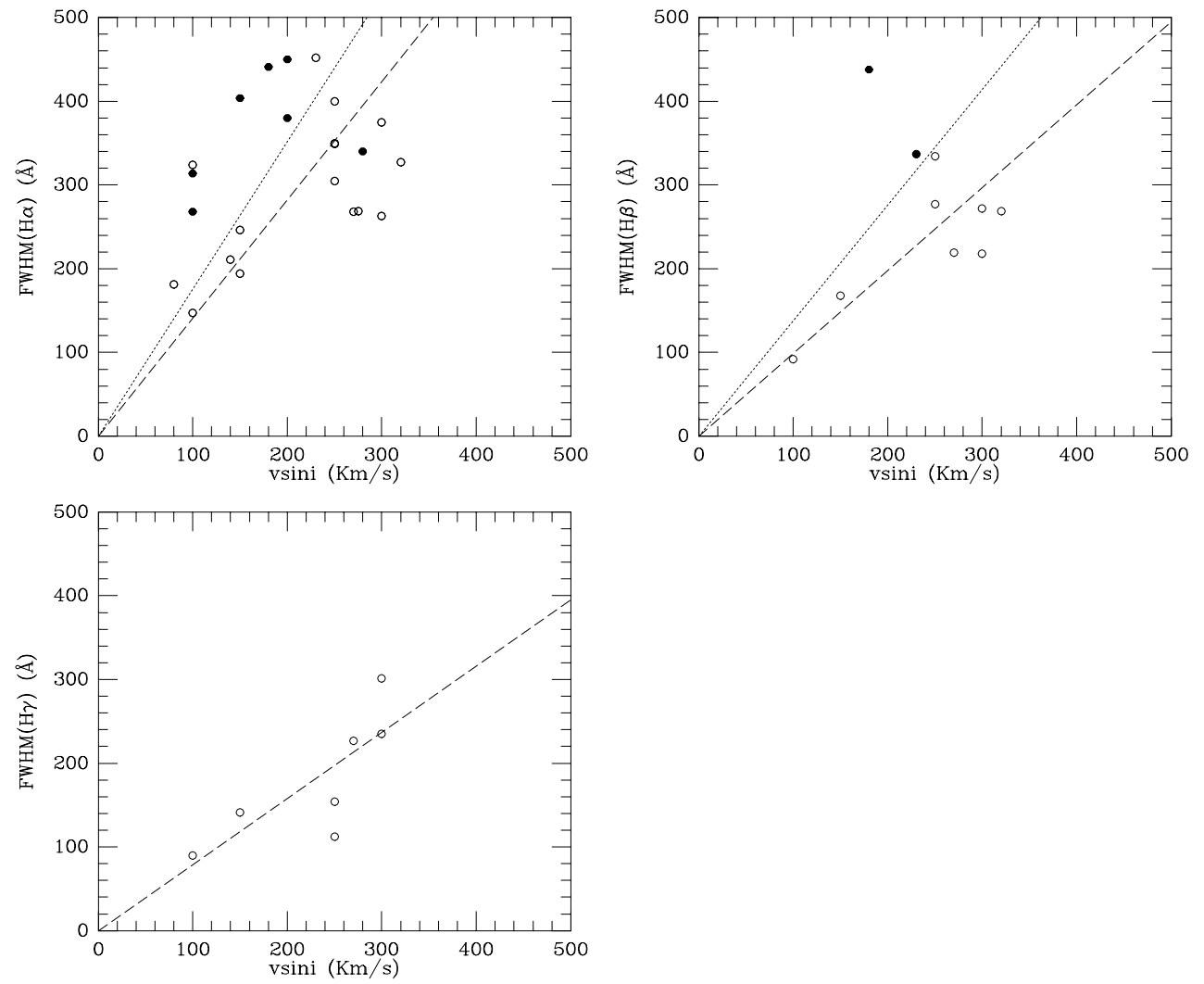

Fig. 16. Full width at half maximun for $\mathrm{H} \alpha, \mathrm{H} \beta$ and $\mathrm{H} \gamma$ emission lines vs. $v \sin i$. Dotted lines represent the relationship for the whole sample while dashed lines represent the relationship for single peaked profiles. Open circles and solid circles represent stars which show single peaked and double peaked profiles respectively in our spectra

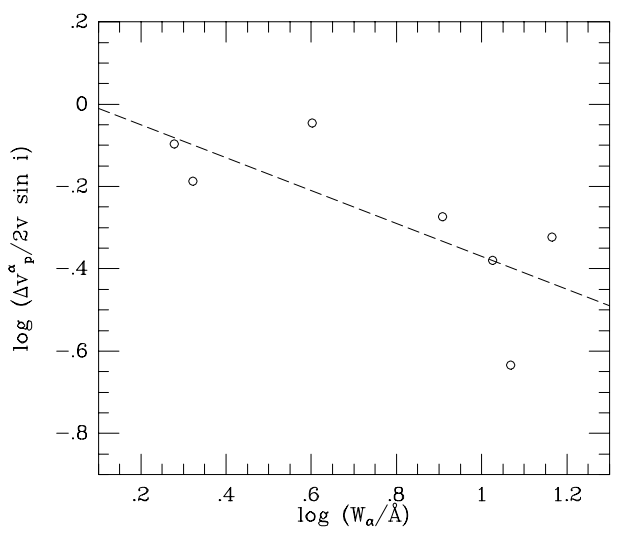

Fig. 17. Velocity peak separation vs. equivalent width for $\mathrm{H} \alpha$ 